chende Mortalitätsrate unter Berücksichtigung beider Zugangswege 2013 noch insgesamt $5,72 \%$, lag sie im folgenden Jahr nur noch bei 4,16\% - nach Einschätzung von Hamm ein „dramatischer Rückgang“.

\section{TAVI auf fast allen}

\section{Risikostufen sicherer}

Eine Analyse des Sterberisikos in Abhängigkeit vom zu erwartenden Operationsrisiko (beurteilt anhand des logistischen Aortenklappenscores 2.0) zeigt, dass das Sterberisiko unmittelbar nach herzchirurgischem Aortenklappener- satz nur bei Patienten mit sehr niedrigem Operationsrisiko etwas geringer war als nach transvaskulärer TAVI-Implantation. Zu berücksichtigen sei dabei jedoch die Tatsache, dass die TAVI-Patienten im Durchschnitt rund zwölf Jahre älter waren. In allen übrigen Risikogruppen schnitten beide Varianten der TAVI besser ab als die Chirurgie.

Dem AQUA-Qualitätsbericht 2014 zufolge lag das Sterberisiko eines Patienten mit sehr geringem Risiko nach einer chirurgischen Klappenimplantation bei $1,08 \%$, im Vergleich zu 1,92\% nach endovaskulärer und 2,43\% nach transapika- ler TAVI. Bereits auf der nächst höheren Risikostufe war die chirurgische Klappenoperation mit einer Sterblichkeit von $4,16 \%$ assoziiert, gegenüber $2,99 \%$ nach endovaskulärer TAVI. Auf der höchsten Risikostufe war die Krankenhaussterblichkeit nach chirurgischer Klappenimplantation mit $27,68 \%$ mehr als doppelt so hoch als nach transapikaler $(13,14 \%)$ und endovaskulärer TAVI (10,72\%).

Auf allen Risikostufen erwies sich der transvaskuläre Zugangsweg sicherer als die Implantation über die transapikale Route.

Peter Overbeck

\title{
Lärm als Hypertonie-Äquivalent
}

Geht die Hälfte aller Herzinfarkte auf schädigende Umweltfaktoren zurück? Zumindest gibt es immer mehr Hinweise, dass insbesondere Lärm nicht nur die Ohren, sondern auch die Blutgefäße in Mitleidenschaft zieht.

n den kardiologischen Risikoscores für Koronarereignisse finden sich Luftverschmutzung und Lärm als relevante Faktoren noch nicht. Und auch die europäischen kardiologischen Leitlinien schweigen sich dazu bisher weitgehend aus: „Nicht einmal in der Präventionsleitlinie der ESC kommt das Wort ,Lärm' vor“, sagte Prof. Thomas Münzel, Mainz. Das könnte sich ändern. Denn es gibt immer mehr Hinweise darauf, dass Lärm das kardiovaskuläre Risiko erhöhen könnte. So hat eine aktuelle Studie aus London in einer detaillierten geografischen Analyse den Zusammenhang zwischen Tages- und Nachtlärm und Gesamtsterblichkeit bzw. kardiovaskulärer Sterblichkeit und kardiovaskulären Ereignissen untersucht.

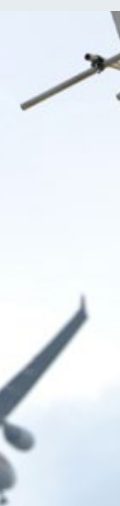

\section{Lärm erhöht das Schlaganfallrisiko}

Im Vergleich zu Straßenlärm unter 55 Dezibel (dB) erhöht Straßenlärm über $60 \mathrm{~dB}$ das Schlaganfallrisiko bei Erwachsenen um signifikante $5 \%$ und bei über 75 -Jährigen um signifikante $9 \%$. Auch Gesamtsterblichkeit, ischämische Herzerkrankungen und kardiovaskuläre Todesereignisse waren teils signifikant erhöht. Münzel berichtete in Berlin über eine eigene Studie, bei der seine Mitarbeiter mithilfe eines MP3-Players Nachtfluglärm simuliert haben, und zwar 30 und 60 Überflüge pro Nacht.

Untersucht wurde, wie sich das auf die Endothelfunktion auswirkt. Anlass der Studie war u.a. eine neue Start- und Landebahn des Frankfurter Flughafens, die dazu führte, dass regelmäßig ab 5 Uhr morgens Flugzeuge mit bis $76 \mathrm{~dB}$ über das Uniklinikum donnern. „Die WHO empfiehlt für Krankenhäuser maximal 55 dB“, so Münzel.

In ihrer Studie mit gesunden Probanden zeigten die Mainzer Kardiologen, dass sich die Endothelfunktion durch den Lärm verschlechtert. „Das war dosisabhängig. Bei 60 Lärmereignissen war der Effekt signifikant stärker“, berichtete Münzel. Eine weitere Studie ergab, dass der Zusammenhang zwischen Lärm und endothelialer Dysfunktion bei KHK-Patienten noch ausgeprägter ist als bei Gesunden. Durch Vitamin C ließ sich die lärminduzierte endotheliale Dysfunktion teils antagonisieren. Die These sei daher, dass Lärm zu oxidativem Stress führe, der wiederum das Endothel schädige, so Münzel.

Philipp Grätzel

\footnotetext{
Literatur

1. Halonen Jl et al.. Eur Heart J 2015; online am 23. Juni 2015; doi: 10.1093/eurheartj/ehv216
} 